\title{
Critical phenomena in 1D Ising model with arbitrary spin
}

\author{
Alexey Proshkin ${ }^{1, \star}$, Felix Kassan-Ogly ${ }^{1, \star \star}$, Alexander Zarubin ${ }^{1}$, Tatyana Ponomareva ${ }^{2}$, and Ivan Menshikh ${ }^{2}$ \\ ${ }^{1}$ Institute of Metal Physics of Ural Branch of Russian Academy of Sciences, 620990, Ekaterinburg, Russia \\ ${ }^{2}$ Ural Federal University, 620002, Ekaterinburg, Russia
}

\begin{abstract}
The aim of this work was to study critical phenomena taking place in 1D Ising model with different exchange interactions signs and arbitrary spin values in a magnetic field. Exact analytical formulas for frustration fields, zero temperature magnetization and entropy at these fields are obtained. The general behavior of pair spin correlation function with the accounting of only interactions between nearest neighbors is examined.
\end{abstract}

\section{Introduction}

Due to recent developments in synthesizing lowdimensional systems there is now a great interest devoted to unique properties of these materials $[1,2]$. Moreover as was shown previously such real systems as lanthanide and actinide monopnictides and monochalcogenides can adequately be described in terms of low-dimensional Ising and Potts models [3-5]. The magnetic and thermodynamic properties of «classical» Ising and Potts models on different lattices were thoroughly investigated in the literature, see, for example, [6-10]. But there is a lack of works in which the accounting of different spin values $s$ is examined. The aim of this work was to study critical phenomena taking place in the Ising model on a linear chain with arbitrary spin values $s$. The Hamiltonian of the system with the accounting of nearest-neighbors $J_{1}$ and nextnearest-neighbors interactions $J_{2}$ in a magnetic field $H$ has a standard form:

$$
\mathcal{H}=-J_{1} \sum_{i} S_{i} S_{i+1}-J_{2} \sum_{i} S_{i} S_{i+2}-H \sum_{i} S_{i},
$$

where $S$ in general takes $2 s+1$ values $-s,-s+1 \ldots s-1, s$.

The investigations were performed by the method of Kramers-Wannier transfer-matrix [11], in which one has to construct transfer-matrix and obtain a maximum eigen value of this matrix. All parameters such as free energy, magnetization and entropy can only be expressed in terms of the maximum eigen value of the corresponding transfermatrix.

In the first chapter of the article we discuss magnetization and entropy peculiarities of the 1D Ising model with arbitrary spin; in the second chapter the behavior of pair spin correlation function in the cases $s=1 / 2$ and $s=1$ is examined and generalizations for the arbitrary spin values are made.

${ }^{\star}$ e-mail: proshkin_ai@imp.uran.ru

$\star \star$ e-mail: felix.kassan-ogly@imp.uran.ru

\section{Magnetization and entropy}

\subsection{Nearest-neighbors in a magnetic field}

It is known that the one-dimensional antiferromagnetic $(J<0$ in Eq. (1)) Ising model with the only accounting of nearest-neighbors interaction possesses frustrations at certain critical magnetic fields at which at low temperatures the dramatic change of the ground state takes place [4]

$$
H_{\mathrm{fr}}=-2 s J
$$

We were able to derive analytical expressions for magnetization and entropy at these frustration fields as functions of $s$ at $T \rightarrow 0$ :

$$
\begin{aligned}
& M_{\mathrm{fr}}=\left(\frac{2 s-1}{4}\right)+\left(\frac{2 s+1}{4}\right) \frac{1}{\sqrt{1+8 s}}, \\
& S_{\mathrm{fr}}=\ln \left(\frac{1+\sqrt{1+8 s}}{2}\right) .
\end{aligned}
$$

Figure 1 demonstrates the example of magnetization and entropy behavior in the one-dimensional antiferromagnetic Ising model with $s=3 / 2$. It is clearly visible that the magnetization at low temperatures at $H=3$ has a jump from $M=0$ to $M=3 / 2$, magnetization at this field at $T \rightarrow 0$ tends to the value $1 / 2+1 / \sqrt{13}$ and the zero-temperature entropy tends to non-zero value $\ln [(1+\sqrt{13}) / 2]$ in all accordance with the equations (2)(4). A small change in the magnetic field results in the zero entropy at $T \rightarrow 0$. Asymptotic value of the entropy at infinite temperature is equal to the natural logarithm of the number of possible states, in the case $s=3 / 2$ it would be $S_{\infty}=\ln 4$.

\subsection{Next-nearest neighbors in the absence of magnetic field}

The accounting of the next-nearest-neighbors with antiferromagnetic exchange leads to the two different ground 


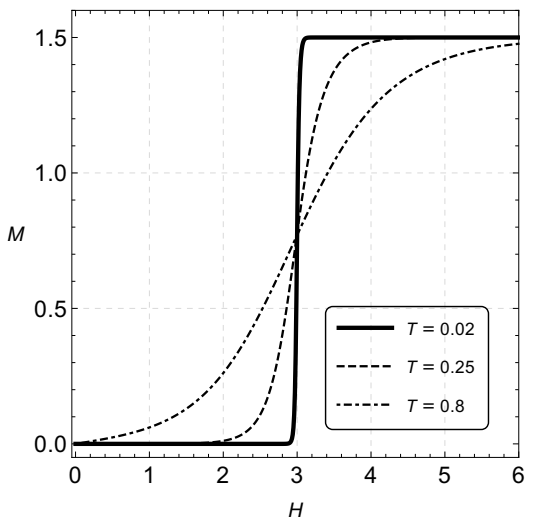

(a)

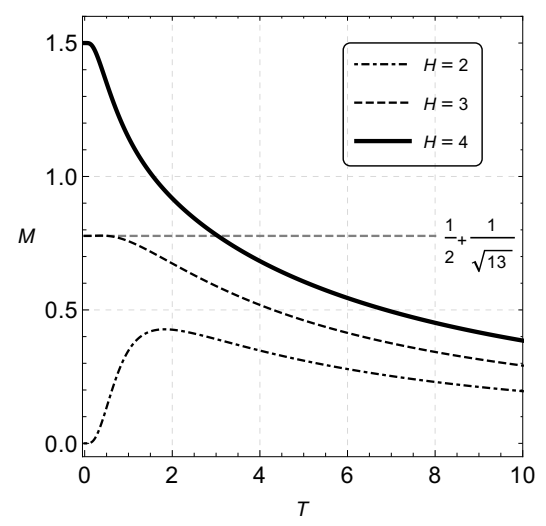

(b)

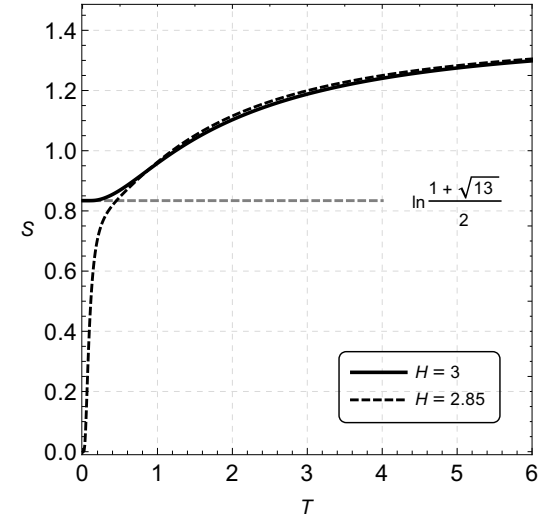

(c)

Figure 1. Magnetization as a function of magnetic field (a) and temperature (b); entropy as a function of temperature (c) in 1D antiferromagnetic Ising model with $s=3 / 2$.

states at $T \rightarrow 0$ depending upon the ratio of $R=J_{2} / J_{1}$. In this case frustrations appear at $|R|=0.5$ for both cases $J_{1}<0, J_{2}<0$ and $J_{1}>0, J_{2}<0$ called competing interactions [12]. It was found that at the frustration point the entropy for both competing interactions at $T \rightarrow 0$ has the same value:

$$
\begin{array}{r}
S_{\mathrm{fr}}=\ln \left\{\frac { 1 } { 3 } \left[1+\left(27 s-8+3 \sqrt{3} \sqrt{27 s^{2}-16 s}\right)+\right.\right. \\
\left.\left.\left(27 s-8-3 \sqrt{3} \sqrt{27 s^{2}-16 s}\right)\right]\right\} .
\end{array}
$$

It is interesting to note that the entropy at $s=1 / 2$ coincides with the entropy derived for the model with the nearest-neighbor-interactions only (suppose $s=1 / 2$ in Eq. (4)) and is equal to natural logarithm of famous golden ratio.

\subsection{Next-nearest neighbors in a magnetic field}

General behavior of this model in the «classical» case is represented in [12]. Here we write generalized equations for the case of arbitrary spin. The case when both exchange interaction values are negative (antiferromagnetic) is assumed. The results for the other case will be published elsewhere.

For both antiferromagnetic interactions there exist two different frustration fields depending upon the ratio $R$ :

$$
\begin{aligned}
& H_{1}= \begin{cases}-2 s J_{1}+4 s J_{2}, & 0<R \leq 0.5 ; \\
s J_{1}-2 s J_{2}, & R \geq 0.5 .\end{cases} \\
& H_{2}=-2 s J_{1}-2 s J_{2} .
\end{aligned}
$$

In Figure 2 the calculated magnetizations for $s=3 / 2$ at $T=0.05$ for five cases of parameter $R$ are shown. The jumps of magnetizations at critical fields determined by the Eq. (6) are clearly pronounced.

Exact analytical expressions for magnetization and entropy at $T \rightarrow 0$ at frustration fields as functions of $s$ are listed in [4].

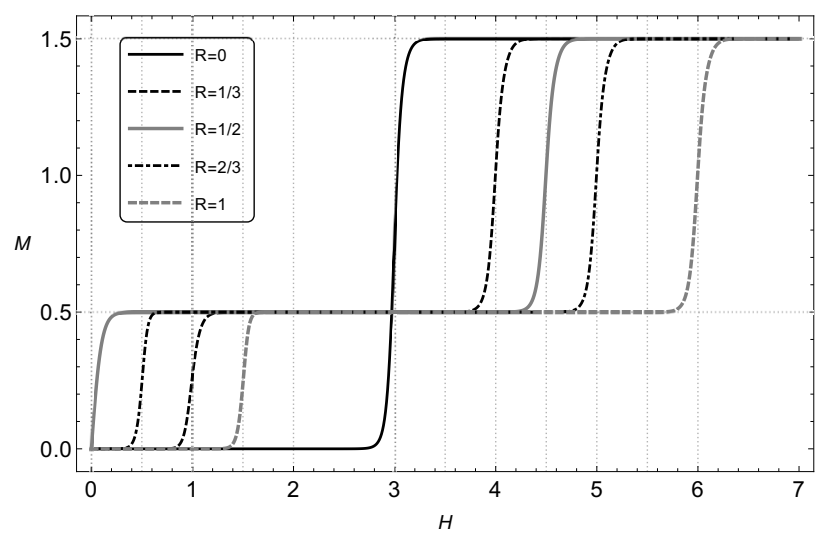

Figure 2. Magnetization of the one-dimensional Ising model with nearest-neighbor and next-nearest-neighbor antiferromagnetic interactions with $s=3 / 2$ at $T=0.05$ for five different values of parameter $R=J_{2} / J_{1}$

\section{Correlation function}

\subsection{Correlation function in the absence of magnetic field}

This chapter is devoted to the behavior of the Fouriertransform of the pair spin correlation function in the onedimensional Ising model with the accounting of interactions between nearest-neighbors in the case $s=1$. Generalizations for the arbitrary spin values $s$ are made at the end of the chapter. The results for the correlation function of the Ising model with allowance of next-nearest-neighbor interactions are to be published.

In the absence of external magnetic field the expression for Fourier-transform of pair spin correlation function with $s=1$ has the form

$$
K(q)=\cos ^{2}\left[\frac{1}{2} \operatorname{arccot}\left(\frac{2 \cosh \frac{J}{T}-1}{2 \sqrt{2}}\right)\right] \frac{1-\eta^{2}}{1-2 \eta \cos q+\eta^{2}},
$$




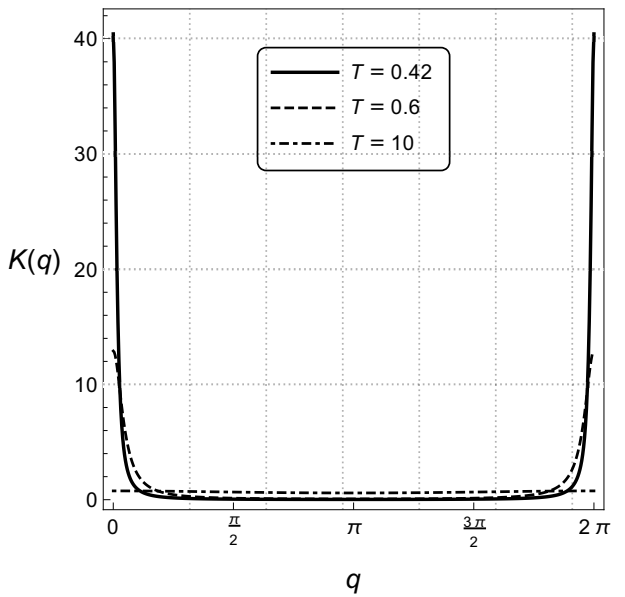

(a)

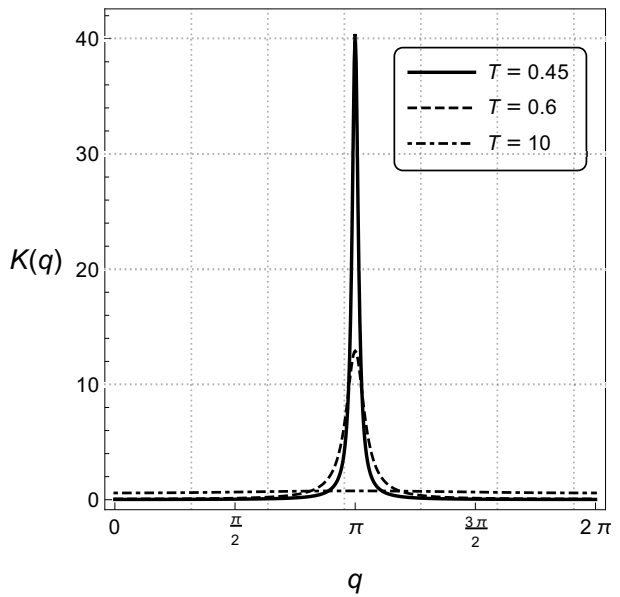

(b)

Figure 3. The dependence of the correlation function upon wave-vector $q$ for the ferromagnetic $(J>0)$ (a) and antiferromagnetic $(J<0)$ interactions (b) in the one-dimensional Ising model with $s=1$ at zero magnetic field.

where $q$ - wave vector, $\eta=\lambda_{2} / \lambda_{1}$ :

$$
\begin{aligned}
& \lambda_{1}=\cosh \frac{J}{T}+\frac{1}{2}+\sqrt{\left(\cosh \frac{J}{T}-\frac{1}{2}\right)^{2}+2}, \\
& \lambda_{2}=2 \sinh \frac{J}{T} .
\end{aligned}
$$

In the ferromagnetic case (Fig. 3a) correlation function has peaks exactly at the positions of Bragg reflections $q_{n}=2 \pi n, n \in \mathbb{Z}$ and at $T \rightarrow 0$ takes the delta-function form what means that the phase-transition takes place (1D systems undergo a phase-transition at zero-temperature).

Figure $3 \mathrm{~b}$ demonstrates the calculation of correlation function in the antiferromagnetic case. It is clearly visible that the peaks are located exactly in the middle of Bragg reflections $q_{n}=\pi(2 n+1), n \in \mathbb{Z}$ because the ground state in this case $(+-+-)$ has doubled translation period compared to the lattice. At $T \rightarrow 0$ correlation function again takes the form of delta-function.

\subsection{Correlation function in a magnetic field}

The expression for the correlation function for the Ising model with $s=1$ in an external magnetic field consists of three terms

$$
K(q)=M^{2} L(q)+X D_{1}(q)+Y D_{2}(q),
$$

where $M$ - magnetization, $L(q)$ - Laue function, $D(q)$ - diffuse scattering function. Let us suppose $k=J / T$ and $h=H / T$, that

$$
\begin{aligned}
& M=\cos ^{2} \beta \cos ^{2} \gamma- \\
& (\cos \alpha \sin \beta \cos \gamma+\sin \alpha \sin \gamma)^{2}, \\
& \tan \alpha=e^{k+h / 2} \frac{\lambda_{3}-2 e^{-h} \sinh k}{1-e^{k}-\lambda_{3}}, \\
& \tan \beta=-e^{h} \frac{\lambda_{3}-2 e^{-h} \sinh k}{\lambda_{3}-2 e^{h} \sinh k} \cos \alpha, \\
& \cos ^{2} \gamma=\frac{1}{\lambda_{1}-\lambda_{2}}\left(e^{k+h}-\lambda_{2}+\tan ^{2} \beta\left(e^{k+h}-\lambda_{3}\right)\right) .
\end{aligned}
$$

The eigenvalues $\lambda\left(\lambda_{1}>\lambda_{2}>\lambda_{3}\right)$ may easily be found from the equation

$$
\begin{aligned}
& \lambda^{3}-\left(1+2 e^{k} \cosh h\right) \lambda^{2}-\left(2 \cosh h\left(1-e^{k}\right)-\right. \\
& 2 \sinh 2 k) \lambda+4 \sinh k-2 \sinh 2 k=0,
\end{aligned}
$$

Laue function $L(q)$ defines positions of Bragg reflections while functions $D(q)$ define diffuse scattering.

$$
\begin{aligned}
L(q) & =\frac{1}{N} \frac{\sin ^{2}(q N / 2)}{\sin ^{2}(q / 2)}, \\
D_{1}(q) & =\frac{1-\eta_{2}^{2}}{1-2 \eta_{2} \cos q+\eta_{2}^{2}}, \\
D_{2}(q) & =\frac{1-\eta_{3}^{2}}{1-2 \eta_{3} \cos q+\eta_{3}^{2}},
\end{aligned}
$$

where $\eta_{2}=\lambda_{2} / \lambda_{1}$ and $\eta_{3}=\lambda_{3} / \lambda_{1}$.

$$
Y=\cos ^{2} \beta\left[\left(1+\cos ^{2} \alpha\right) \sin \beta \cos \gamma+\frac{1}{2} \sin 2 \alpha \sin \gamma\right]
$$

and all coefficients in (10) are connected as follows:

$$
X+Y+M^{2}=\left\langle S^{2}\right\rangle,
$$

where $\left\langle S^{2}\right\rangle$ is the mean squared value of the spin:

$$
\begin{aligned}
& \left\langle S^{2}\right\rangle=\cos ^{2} \beta \cos ^{2} \gamma+ \\
& (\cos \alpha \sin \beta \cos \gamma+\sin \alpha \sin \gamma)^{2} .
\end{aligned}
$$

It is interesting to compare Eq. (11) and Eq. (19).

Laue function defines Bragg scattering, does not depend upon exchange interaction sign and is characterized by lattice parameters. Ferromagnetic system does not possess frustrations and $D(q)=X D_{1}(q)+Y D_{2}(q)$ in this case is equal to zero at low temperatures. Thus hereinafter we will focus on a behavior of the diffuse scattering part of the correlation function (10) in the antiferromagnetic case. 


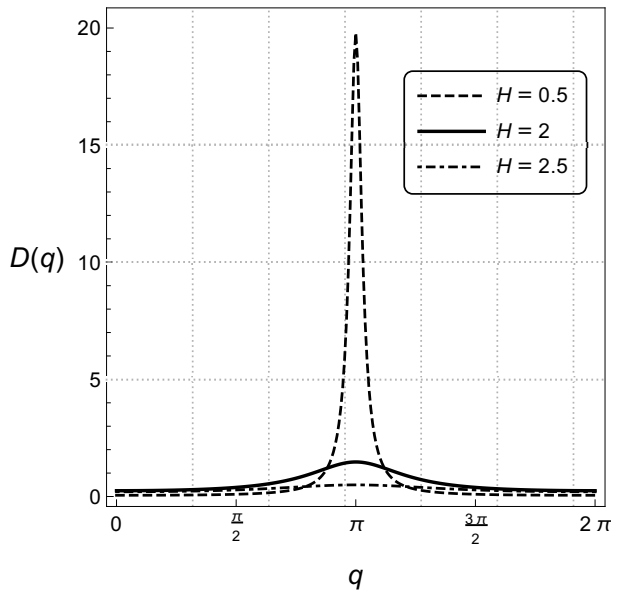

Figure 4. Diffuse scattering in the one-dimensional antiferromagnetic Ising model with $s=1$ at $T=0.5$ at three values of magnetic field $H$.

Figure 4 illustrates the dependence of the diffuse scattering function $D(q)$ upon wave vector $q$ in the onedimensional antiferromagnetic Ising model with $s=1$ at $T=0.5$ for three different values of magnetic field $H$. It is clearly pronounced that above the frustration field $(H>2)$ diffuse scattering vanishes (both coefficients $X$ and $Y$ in $D(q)$ are equal to zero). Below the frustration field $(H<2)$ the function tends to take the delta-function form that says about the phase transition. Exactly at the frustration field $(H=2)$ the function has a broad maximum and does not tend to take the delta-function form at $T \rightarrow 0$. This unusual behavior of the correlation function is the clear evidence that there is no long-range order in the system even at zero temperature. Similar results for the diffuse scattering function were obtained in three- and four-state Potts models [13, 14] and for «classical» Ising model [15]. In the case $s=1 / 2$ the analytical expression for the diffuse scattering function has a simple form:

$$
D(q)=\left(\frac{1}{4}-M^{2}\right) \frac{1-\eta^{2}}{1-2 \eta \cos q+\eta^{2}},
$$

where again $\eta=\lambda_{2} / \lambda_{1}$,

$$
\begin{aligned}
\lambda_{1,2} & =e^{\frac{J}{4 T}}\left(\cosh \frac{H}{2 T} \pm \sqrt{\sinh ^{2} \frac{H}{2 T}+e^{-\frac{J}{T}}}\right), \\
M & =\frac{\sinh \frac{H}{2 T}}{2 \sqrt{\sinh ^{2} \frac{H}{2 T}+e^{-\frac{J}{T}}}} .
\end{aligned}
$$

Since in the case $s=1 / 2$ the asymptotic value of magnetization is equal to $1 / 2$ it is clearly visible from the Eq. (20) that $D(q) \rightarrow 0$ at $M \rightarrow 1 / 2$. The only general difference in the diffuse scattering function behavior between the cases $s=1 / 2$ Eq. (20) and $s=1$ Eq. (10) consists in the different values of frustration field (Eq. (2)). Generally the behavior of the correlation function described above is similar in the one-dimensional antiferromagnetic Ising model for any value of $s$.
It is evident that frustrated states have peculiar properties. Magnetization at frustration fields at low temperatures has jumps, entropy tends to nonzero values and diffuse scattering function has a broad maximum and does not take the form of delta-function.

\section{Summary}

In this article the main results obtained for the onedimensional Ising model are presented. Exact analytical formulas for frustration fields, zero temperature magnetization and entropy at these fields are obtained. The general behavior of pair spin correlation function with the accounting of interactions between nearest neighbors is examined. It is shown that in the antiferromagnetic Ising model a diffuse scattering function at $T \rightarrow 0$ does not take the deltafunction form that is a clear evidence of the absence of the long-range order in the system.

The reported study was carried out within the state assignment of FASO of Russia (theme «Quantum» n. 01201463332) and was funded by RFBR according to the research project n. 16-32-00032.

\section{References}

[1] T. Giamarchi, Quantum physics in one dimension (Oxford: Oxford university press, 2003)

[2] S. Roth, D. Carroll, One-dimensional metals: conjugated polymers, organic crystals, carbon nanotubes and graphene (Wiley-VCH, 2015)

[3] F.Y. Wu, Rev. Mod. Phys. 54, 235 (1982)

[4] A. Proshkin, F. Kassan-Ogly, Mater. Sci. Forum 845, 93 (2016)

[5] F.A. Kassan-Ogly, A.K. Murtazaev, A.K. Zhuravlev, M.K. Ramazanov, A.I. Proshkin, J. Magn. Magn. Mater. 384, 247 (2015)

[6] L. Onsager, Phys. Rev. 65, 117 (1944)

[7] A.K. Murtazaev, M.K. Ramazanov, M.K. Badiev, Low Temp. Phys. 37, 1001 (2011)

[8] F.A. Kassan-Ogly, B.N. Filippov, A.K. Murtazaev, M.K. Ramazanov, M.K. Badiev, J. Magn. Magn. Mater. 324, 3418 (2012)

[9] A.I. Proshkin, F.A. Kassan-Ogly, J. Magn. Magn. Mater. 383, 13 (2015)

[10] D.P. Landau, Phys. Rev. B 21, 1285 (1980)

[11] H.A. Kramers, G.H. Wannier, Phys. Rev. 60, 252 (1941)

[12] F.A. Kassan-Ogly, Phase Transitions 74, 353 (2001)

[13] F.A. Kassan-Ogly, B.N. Filippov, Phys. Met. Metallogr. 95, 518 (2003)

[14] F.A. Kassan-Ogly, B.N. Filippov, Phase Transitions 77, 261 (2004)

[15] R.J. Baxter, Exactly solved models in statistical mechanics (Academic press, 1989) 